\title{
IMPROVEMENT THE PROPERTIES OF YOGHURT USING LEGUMES TO THERAPY PROTEIN ENERGY MALNUTRITION
}

\author{
Abtsam M.F. Badr ${ }^{(1)}$, D.A.M. Amer ${ }^{(1)}$, M.Y.A. El-Hawary ${ }^{(1)}$ and A.M.A.Naem ${ }^{(2)}$ \\ (1) Department of Food science and Technology, Faculty of Agriculture, Tanta University, \\ Egypt \\ (2) Department of Pediatrics, Faculty of Medecine , Tanta University, Egypt
}

Received: May. 8, 2018

Accepted: May. 23,2018

\begin{abstract}
The aim of this study was to improve the properties of yoghurt using different kinds of legumes such as chickpea $(0.0,3.0$ and $6.0 \% \mathrm{w} / \mathrm{w})$, kidney bean $(0.0$, 3.0 and $6.0 \% \mathrm{w} / \mathrm{w})$ and cowpea $(0.0,3.0$ and $6.0 \% \mathrm{w} / \mathrm{w})$ in order to therapy protein energy malnutrition in children. Chemical composition, $\mathrm{pH}$, texture, viscosity, and sensory evaluation were determined for all treatments. Results showed that total protein increased in yoghurt treatments as a result of adding legumes compared with control. Yoghurt produced using 6\% kidney beans (T2b) had highest total protein compared with other treatments. Yoghurt produced using cowpea 6\% (T3a) had the higher total solids. Yoghurt produced with $6 \%$ kidney bean (T2b) and produced using $6 \%$ cowpea had the lowest fat / dry matter as compared with other treatment. There were no significant differences in $\mathrm{pH}$ values among all treatments. Also results showed that for all treatments as the rate of legumes increased the viscosity value also increased. The highest viscosity level was recorded with adding cowpea at rate $6 \%$ (T3b). Texture parameters hardness, adhesiveness, cohesiveness, springiness, chewiness and gumminess increased with adding all type of legumes. Yoghurt produced with $6 \%$ kidney bean and chickpea 3\% had improved rheological and organoleptic Properties compared with other treatments.
\end{abstract}

Key words: Malnutrition, Protein-energy malnutrition, legumes and yoghurt.

\section{INTRODUCTION}

Malnutrition is globally the most important risk factor for illnesses and death, affecting especially hundreds of millions of pregnant women and young children. It is currently the leading cause of global burden of disease (Ezzati et al., 2002). The World Health Organization has defined malnutrition as 'the cellular disparity amid the supply of energy, nutrients and the body's demand for them to ascertain maintenance, growth and specific functions (Anstead et al., 2001; Dean et al., 2003 and Rizwana et al., 2015).

Protein-energy malnutrition is defined as a range of pathological conditions arising from a lack of adequate protein and calories (Ernest et al., 2013). It is a problem in many developing countries, of which African countries are mostly affected, in children between the ages of
6 months and 5 years. This type of malnutrition presents itself in the form of kwashiorkor, marasmus and marasmickwashiorkor (Ernest et al., 2013 and Une \& Gupta, 2013). The dietary management of moderate acute malnutrition should normally be based on the optimal use of locally available nutrient-dense foods to improve the nutritional status of children and prevent them from becoming severely acutely malnourished (Ashworth and Ferguson, 2009).

Legumes are higher in protein than any other food plant with values ranging from $17 \%$ to $31 \%$ and the average about $25 \%$. Legumes are close to animal meat in quality and low-cost dietary vegetable proteins and minerals when compared with animal products. (Adeyeba, 2014). Legumes are featured by their high nutritional value and can be recognized and labeled as both a source of 
vegetables and a source of protein. They are especially characterized as a good source of protein and are compared with meat, fish and eggs. They are a relatively cheaper than meat products which make them a good protein-rich alternative regardless of socioeconomic status. (Abrahamson et al., 2006).

Legumes can be a valuable source of energy. The high carbohydrate content contributes a great deal to the energy supply of pulses. The energy content of most pulses has been found to be between 300 and $540 \mathrm{Kcal} / 100 \mathrm{~g}$. Energy is required for all metabolic processes. The energy of Pulses comes from the nutrient supply of protein, fat and carbohydrate for example Cowpeas 340 $\mathrm{kcal} / 100 \mathrm{~g}$ and Chickpeas $347 \mathrm{kcal} / 100 \mathrm{~g}$ (Reddy et al., 1985 and Oke et al., 1995).

Yoghurt is defined by the Codex Alimentations of 2003 as a coagulated milk product that results from the fermentation of milk by streptococcus thermophilus and lactobacillus delbrueckii spp. bulgaricus (CODEX STAN 243-2003). As starter culture for yoghurt production, lactic acid bacterial species display symbiotic relations during their growth in milk medium (Tamime and Robinson, 1999). Yoghurt is a nutrient-dense food that meets a wide variety of nutritional needs at for everyone. It is a rich source of milk proteins, carbohydrate, minerals such as calcium and phosphorous, and vitamins such as riboflavin (B2), thiamin (B1), coalmine (B12), folate (B9), niacin (B3) and vitamin A (Mckinley, 2005).

The objective of this study was to use formulate energy dense yoghurt based weaning food rich in nutrition by supplementation with cow pea, kidney bean and chickpea powder and evaluate the effect of legumes powder addition on microbiological, physicochemical, and sensory of yoghurt.

\section{MATERIALS AND METHODS}

\section{Materials}

Fresh full-fat cow milk was obtained

from local market. The composition of raw milk was as follows: fat $3 \%$, protein $2.90 \%$, total solids $11.9 \%$, acidity was $0.19 \%$ and $\mathrm{pH}$ was 6.64 .

Starter cultures LAB. (Express 0.2, thermophilic yoghurt culture Yo-Flex Express), consisting of Lactobacillus delbrueckii ssp. bulgaricus and Streptococcus thermophilus for manufacture of yoghurt, was obtained from Chr. Hansen's Lab., Denmark.

\section{Legumes: Three types of legumes} were used:

Chickpea Cicerarie thinum, (protein $20.47 \%$, fat $6.04 \%$, fiber $3.5 \%$ and carbohydrate $27.42 \%$ ) , kidney bean Phaseolus vulgaris L., (protein 28.7\%, fiber $6.4 \%$ and fat $0.5 \%$ and carbohydrate $22.8 \%)$ and Cowpea Vignaun guiculata, (protein $24 \%$, fat 1.5 $\%$, fiber $6.6 \%$ and carbohydrate $27.3 \%$ ) were obtained from local market. The seeds were thoroughly cleaned from dust and other extraneous materials prior to use. (Bravo et al., 1999)

\section{Methods}

2.1 Preparation of legumes: The whole legumes of Chickpea, Cowpea and kidney bean were soaked in distilled water $(1: 10 \mathrm{w} / \mathrm{v})$ for $24 \mathrm{~h}$ at room temperature $\left(25{ }^{\circ} \mathrm{C}\right)$. Hulls were removed manually after soaking the seeds according to El-Beltagy, (1996). Seeds were placed in a Birex pot with distilled water $(1: 10 \mathrm{w} / \mathrm{v})$, then cooked in a microwave oven (Sumsung 44L-900W) on high for 15 min (the seeds were soft when felt between the fingers). Beans were oven-dried at $80{ }^{\circ} \mathrm{C}$ for $24 \mathrm{~h}$ to constant weight. The dried seeds were milled into flour using laboratory grinding machine (poly 
mix PX-MFC 90D, Switzerland) and stored in airtight plastic container at $4{ }^{\circ} \mathrm{C}$ until use.

2.2 Manufacture of yoghurt: Fresh full cow's milk was supplemented with 0 , 3 and $6 \%$ legumes powder respectively which were added individually. The control was full fat milk ( $3 \%$ fat, $13.2 \%$ TS) without any additives. Chickpea, cow pea and kidney bean powder were added at 3 and $6 \%$ individually. Yoghurt was manufactured according to the protocol proposed by Tamime and Robinson, (1999).

Individual milk samples were heat treated at $90^{\circ} \mathrm{C}$ for $10 \mathrm{~min}$, cooled to $42^{\circ} \mathrm{C}$ and inoculated with yoghurt culture at the rate recommended by suppliers, Incubation was done at $42^{\circ} \mathrm{C}$ till the $\mathrm{pH}$ reaching 4.9. This was followed by fast cooling to $7^{\circ} \mathrm{C}$ keeping the product at the same temperature overnight to represent fresh samples yoghurt was stored at $7 \pm 1^{\circ} \mathrm{C}$ for 14 days.

\section{Methods of analysis:}

Sampling: Yoghurt samples were taken when (fresh and at 7 and 14 days).

\section{Chemical analysis:}

All samples were analyzed for fat and total protein and dry matter according to A.O.A.C. (2000). And for pH according to Ling (1963).

\section{Viscosity:}

Viscosity was measured using oscillatory viscometer (VR 3000M YR viscometers, Spain), using spindle 4 at speed of 200 r.p.m at $10^{\circ} \mathrm{C}$.(Lal et al., 2006)

\section{Texture analysis:}

Textural properties of yoghurt were evaluated using a texture analyzer (FTC TMS-Pro), USA). Yoghurt samples were evaluated in their cups. Hardness, cohesiveness, springiness and chewiness were evaluated in triplicate as described by Szczesniak et al., (1963) and Bourne, (1978).

\section{Sensory evaluation:}

The samples were assessed for colour, flavour, viscosity, taste and overall acceptability using a nine-point hedonic scale, where 9 indicated "like extremely" and 1 indicated "dislike extremely". Each panelist was provided with enough privacy to avoid biased assessment (Makanjuola, 2012).

\section{Statistical analysis:}

Results were expressed as mean \pm standard deviation (SD) and least significant differences (LSD) for replicates and subjected to Costat, 6.4 $(1998 / 2008)$ that was done to determine the degree of significant among treatments and within storage period.

\section{RESULTS AND DISCUSSION}

\section{Chemical analysis:}

Data in Table (1) present the chemical composition including total protein content (T.P), fat content / dry matter ( $F$ / DM) and total solids (T.S) of yoghurt as affected by adding different legumes at different ratio when fresh. The obtained results showed that T.P\% increased in yoghurt treatments as a result of adding legumes compared with control. These results are in agreement with Zare et al., (2011) who mentioned that legumes are high alternative sources of protein compared with other types of plants. Yoghurt produced using $6 \%$ kidney beans (T2b) had higher T.P. \% compared with other yoghurt treatments. This result is in agreement with Rehman \& Shah (2004) and Yin et al., (2008) who showed that kidney beans had highest content in energy, proteins, carbohydrates minerals and vitamins of the pulse fraction.

Total solids (T.S.) \% increased as the ratio of legumes increased in all treatments compared to control. Yoghurt produced using cowpea (T3a) had the 
higher T.S\% compared with all other treatments.

Results also showed that yoghurt produced using kidney beans $3 \%$ and cow pea $6 \%$ had the lowest F / DM \% compared with other yoghurt treatments and control. Addition of legumes caused significant differences in $F / D M \%$ in all treatments compared with control. Yoghurt produced with $6 \%$ kidney bean (T2b) and produce using 6\% cow pea had the lowest $F / D M \%$ as compared with other treatment.

\section{2. $\mathrm{pH}$ values}

Data in Table (2) describe the changes

in $\mathrm{pH}$ values of control yoghurt and treatments produced using legumes in fresh and during storage. Legumes were not markedly affected $\mathrm{pH}$ values in all treatments compared with control when fresh while at the end of storage period $\mathrm{pH}$ values were significantly decreased in all treatments.

Table (1): Effect of type and ratio of legumes on chemical properties of set yoghurt

\begin{tabular}{|l|c|c|c|}
\hline *Treatments & (T.P.)\% & (T.S.)\% & F\DM\% \\
\hline C1 & $3.18 \pm 0.04$ & $12.07 \pm 0.24$ & $25 \pm 0.01$ \\
\hline T1a & $3.94 \pm 0.02$ & $14.66 \pm 0.06$ & $26 \pm 0.01$ \\
\hline T1b & $4.38 \pm 0.04$ & $16.80 \pm 0.08$ & $24 \pm 0.00$ \\
\hline T2a & $3.84 \pm 0.08$ & $14.53 \pm 0.04$ & $23 \pm 0.00$ \\
\hline T2b & $4.55 \pm 0.13$ & $16.89 \pm 0.03$ & $21 \pm 0.01$ \\
\hline T3a & $3.74 \pm 0.02$ & $14.85 \pm 0.10$ & $23 \pm 0.00$ \\
\hline T3b & $3.92 \pm 0.66$ & $17.03 \pm 0.07$ & $21 \pm 0.00$ \\
\hline LSD & 0.61 & 0.26 & 0.01 \\
\hline
\end{tabular}

Data are Mean \pm S.D., LSD (0.05)

* C: control yoghurt made from full fat cow's milk.T1a: yoghurt made from full fat cow's milk $+3 \%$ chickpea.

T1b: yoghurt made from full fat cow's milk $+6 \%$ chickpea.T2a: yoghurt made from full fat cow's milk $+3 \%$ kidney bean.

T2b: yoghurt made from full fat cow's milk $+6 \%$ kidney bean. T3a: yoghurt made from full fat cow's milk $+3 \%$ cowpea.

T3b: yoghurt made from full fat cow's milk $+6 \%$ cowpea.

Table (2): Effect of type and ratio of legumes on $\mathrm{pH}$ values of set yoghurt during storage period

\begin{tabular}{|l|c|c|c|c|c|}
\hline \multirow{2}{*}{ *Treatments } & \multicolumn{3}{|c|}{ Storage period } & \multirow{2}{*}{ LSD } \\
\cline { 2 - 5 } & Fresh & 7 days & 14 days & Mean & LS \\
\hline C1 & $4.71 \pm 0.01$ & $4.41 \pm 0.01$ & $4.32 \pm 0.01$ & 4.48 & - \\
\hline T1a & $4.52 \pm 0.01$ & $4.29 \pm 0.05$ & $4.20 \pm 0.08$ & 4.33 & \\
\hline T1b & $4.60 \pm 0.10$ & $4.41 \pm 0.08$ & $4.30 \pm 0.07$ & 4.46 & \\
\hline T2a & $4.55 \pm 0.03$ & $4.33 \pm 0.02$ & $4.18 \pm 0.04$ & 4.35 & \\
\hline T2b & $4.60 \pm 0.04$ & $4.32 \pm 0.07$ & $4.21 \pm 0.05$ & 4.37 & \\
\hline T3a & $4.61 \pm 0.04$ & $4.32 \pm 0.13$ & $2.65 \pm 2.16$ & 3.86 & \\
\hline T3b & $4.58 \pm 0.01$ & $4.35 \pm 0.04$ & $4.17 \pm 0.06$ & 4.36 & \\
\hline
\end{tabular}


Improvement the properties of yoghurt using legumes to therapy

\begin{tabular}{|l|c|c|c|}
\hline LSD & \multicolumn{3}{|c|}{-} \\
\hline Mean & 4.61 & 4.34 & 4.00 \\
\hline LSD & \multicolumn{3}{|c|}{0.27} \\
\hline
\end{tabular}

See legend to table (1) for details.

\section{Viscosity}

Significant variation was noted in viscosities of yoghurt from different treatments produced by adding legumes. Results in Table (3) showed that the highest viscosity level was recorded with adding cowpea at rate $6 \%$ (T3b). For all treatments as the rate of legumes increased the viscosity value also increased. The increase of viscosity may be due to the interaction between the legumes and protein particles thus contributing a strong gel when the concentration was doubled.

\section{Texture properties}

Rheological properties for foods, such as fermented dairy products, are important in the design of flow processes, quality control, storage and processing and in predicting the texture of food (shaker et al., 2000). The hardness of yoghurt is directly dependent on its total solids and specifically protein content and the type of proteins. Higher protein content would cause a higher degree of cross-linkage of the gel network, resulting in a much denser and more rigid gel structure (Tamime, 2006). Table (4) showed texture parameters (Hardness, Adhesiveness, Cohesiveness, Springiness, Chewiness and Gumminess) of the different treatment of yoghurt over storage. Texture parameters were increased with adding all type of legumes. Higher concentration of all legumes increased fracturability and firmness in samples when compared with low level and control. These results are agreement with (Sandoval-castilla et al., 2004) who illustrated that legumes flour could be potentially consider as texture improvement ingredient for yoghurt supplementation.

Table (3): Effect of type and ratio of legumes on viscosity value of set yoghurt during storage period

\begin{tabular}{|l|l|l|l|l|l|}
\hline \multirow{2}{*}{ *Treatments } & \multicolumn{2}{|l|}{ Storage period } & Mean & \multirow{2}{*}{ LSD } \\
\cline { 2 - 5 } & Fresh & 7 days & 14 days & & \multirow{2}{*}{ (202.75 } \\
\hline C1 & $6789.0 \pm 83.44$ & $7022.0 \pm 186.68$ & $7359.5 \pm 183.14$ & 7056.83 & \\
\hline T1a & $8675.0 \pm 35.36$ & $9096.0 \pm 166.88$ & $9289.5 \pm 34.65$ & 9020.17 & \\
\hline T1b & $11066.5 \pm 265.17$ & $11791.0 \pm 9.90$ & $12144.5 \pm 0.71$ & 11667.33 & \\
\hline T2a & $9284.0 \pm 98.99$ & $9962.0 \pm 117.38$ & $10287.0 \pm 233.35$ & 9844.33 & \\
\hline T2b & $12029.5 \pm 7.78$ & $12401.0 \pm 79.20$ & $12676.0 \pm 280.01$ & 12368.83 & \\
\hline T3a & $9261.0 \pm 84.85$ & $10061.5 \pm 259.51$ & $11116.0 \pm 182.43$ & 10146.17 & \\
\hline T3b & $11666.0 \pm 690.14$ & $12319.0 \pm 596.80$ & $12814.5 \pm 180.31$ & 12266.50 & \\
\hline
\end{tabular}


Abtsam M.F. Badr

\begin{tabular}{|l|l|l|l|l|}
\hline LSD & \multicolumn{4}{|l|}{} \\
\hline Mean & 9824.43 & 10378.93 & 10812.43 & \\
\hline LSD & 143.37 & & \\
\hline
\end{tabular}

Table (4): Effect of type and ratio of legumes on Texture properties of set yoghurt during storage period

\begin{tabular}{|c|c|c|c|c|c|c|c|c|c|c|}
\hline \multirow{2}{*}{$\begin{array}{c}\text { Texture } \\
\text { properties }\end{array}$} & \multirow{2}{*}{$\begin{array}{c}\text { Storage } \\
\text { period }\end{array}$} & \multicolumn{7}{|c|}{ "Treatments } & \multirow[b]{2}{*}{ Mean } & \multirow[b]{2}{*}{ LSD } \\
\hline & & C & T1a & T1b & T2a & T2b & T3a & T3b & & \\
\hline \multirow[t]{3}{*}{$\begin{array}{c}\text { Hardness } \\
\text { (g) }\end{array}$} & Fresh & $\begin{array}{c}98.50 \pm \\
4.95\end{array}$ & $\begin{array}{c}135.00 \pm \\
14.14\end{array}$ & $\begin{array}{c}215.00 \pm \\
7.07\end{array}$ & $\begin{array}{c}159.50 \pm \\
7.78\end{array}$ & $\begin{array}{c}230.00 \pm \\
21.21\end{array}$ & $\begin{array}{c}145.00 \pm \\
0.00\end{array}$ & $\begin{array}{c}247.50 \pm \\
9.19\end{array}$ & 175.79 & \multirow{3}{*}{6.88} \\
\hline & 7 days & $\begin{array}{c}111.00 \pm \\
4.24\end{array}$ & $\begin{array}{c}155.00 \pm \\
14.14\end{array}$ & $\begin{array}{c}265.50 \pm \\
13.44\end{array}$ & $\begin{array}{c}182.50 \pm \\
21.92\end{array}$ & $\begin{array}{c}254.50 \pm \\
13.44 \\
\end{array}$ & $\begin{array}{c}170.50 \pm \\
4.95\end{array}$ & $\begin{array}{c}276.00 \pm \\
15.56\end{array}$ & 202.14 & \\
\hline & 14 days & $\begin{array}{c}122.50 \pm \\
2.12\end{array}$ & $\begin{array}{c}177.00 \pm \\
14.14\end{array}$ & $\begin{array}{c}309.00 \pm \\
15.56\end{array}$ & $\begin{array}{c}201.00 \pm \\
12.73\end{array}$ & $\begin{array}{c}288.00 \pm \\
14.14\end{array}$ & $\begin{array}{c}189.50 \pm \\
3.54\end{array}$ & $\begin{array}{c}305.00 \pm \\
0.00\end{array}$ & 227.43 & \\
\hline \multicolumn{2}{|c|}{ Mean } & 110.67 & 155.67 & 263.17 & 181.00 & 257.50 & 168.33 & \multicolumn{2}{|c|}{276.17} & \multirow{5}{*}{4.86} \\
\hline \multicolumn{2}{|c|}{ LSD } & \multicolumn{8}{|c|}{9.72} & \\
\hline \multirow{3}{*}{$\begin{array}{c}\text { Adhesiven } \\
\text { ess } \\
\text { (g.mm) }\end{array}$} & Fresh & $\begin{array}{c}65.50 \pm \\
3.54\end{array}$ & $\begin{array}{c}113.00 \pm \\
1.41\end{array}$ & $\begin{array}{c}141.50 \pm \\
4.95\end{array}$ & $\begin{array}{c}119.50 \pm \\
3.54\end{array}$ & $\begin{array}{c}129.50 \pm \\
6.36\end{array}$ & $\begin{array}{c}94.50 \pm \\
4.95\end{array}$ & $\begin{array}{c}144.50 \pm \\
13.44\end{array}$ & 115.43 & \\
\hline & 7 days & $\begin{array}{c}73.00 \pm \\
2.83\end{array}$ & $\begin{array}{c}121.50 \pm \\
3.54\end{array}$ & $\begin{array}{c}160.50 \pm \\
6.36\end{array}$ & $\begin{array}{c}125.50 \pm \\
9.19\end{array}$ & $\begin{array}{c}141.50 \pm \\
10.61\end{array}$ & $\begin{array}{c}103.00 \pm \\
1.41\end{array}$ & $\begin{array}{c}161.50 \pm \\
23.33\end{array}$ & 126.64 & \\
\hline & 14 days & $\begin{array}{c}75.00 \pm \\
4.24\end{array}$ & $\begin{array}{c}127.50 \pm \\
6.36\end{array}$ & $\begin{array}{c}169.50 \pm \\
6.36\end{array}$ & $\begin{array}{c}138.50 \pm \\
9.19\end{array}$ & $\begin{array}{c}159.00 \pm \\
4.24\end{array}$ & $\begin{array}{c}105.00 \pm \\
1.41\end{array}$ & $\begin{array}{c}175.00 \pm \\
14.14\end{array}$ & 135.64 & \\
\hline \multicolumn{2}{|c|}{ Mean } & 71.17 & 120.67 & 1157.17 & 127.83 & 143.33 & 100.83 & \multicolumn{2}{|c|}{160.33} & \\
\hline \multicolumn{2}{|c|}{ LSD } & \multicolumn{8}{|c|}{6.87} & \\
\hline \multirow[t]{3}{*}{$\begin{array}{c}\text { Cohesiven } \\
\text { ess }\end{array}$} & Fresh & $\begin{array}{c}0.31 \pm \\
0.02 \\
\end{array}$ & $\begin{array}{c}0.39 \pm \\
0.05 \\
\end{array}$ & $\begin{array}{c}0.43 \pm \\
0.02 \\
\end{array}$ & $\begin{array}{c}0.44 \pm \\
0.01 \\
\end{array}$ & $\begin{array}{c}0.45 \pm \\
0.04 \\
\end{array}$ & $\begin{array}{c}0.42 \pm \\
0.00 \\
\end{array}$ & $\begin{array}{c}0.45 \pm \\
0.01 \\
\end{array}$ & 0.41 & \\
\hline & 7 days & $\begin{array}{c}0.33 \pm \\
0.02\end{array}$ & $\begin{array}{c}0.42 \pm \\
0.01\end{array}$ & $\begin{array}{c}0.44 \pm \\
0.02\end{array}$ & $\begin{array}{c}0.45 \pm \\
0.00\end{array}$ & $\begin{array}{c}0.46 \pm \\
0.04\end{array}$ & $\begin{array}{c}0.44 \pm \\
0.03\end{array}$ & $\begin{array}{c}0.46 \pm \\
0.02\end{array}$ & 0.43 & \\
\hline & 14 days & $\begin{array}{c}0.34 \pm \\
0.01\end{array}$ & $\begin{array}{c}0.43 \pm \\
0.01\end{array}$ & $\begin{array}{c}0.45 \pm \\
0.02\end{array}$ & $\begin{array}{c}0.45 \pm \\
0.01\end{array}$ & $\begin{array}{c}0.45 \pm \\
0.06\end{array}$ & $\begin{array}{c}0.44 \pm \\
0.01\end{array}$ & $\begin{array}{c}0.46 \pm \\
0.01\end{array}$ & 0.43 & \\
\hline \multicolumn{2}{|c|}{ Mean } & 0.32 & 0.41 & 00.44 & 0.45 & 0.45 & 0.43 & 0.45 & & \\
\hline \multicolumn{2}{|c|}{ LSD } & \multicolumn{8}{|c|}{$\longrightarrow$} & \\
\hline \multirow[t]{3}{*}{$\begin{array}{c}\text { Springines } \\
\text { s (m.m) }\end{array}$} & Fresh & $\begin{array}{c}0.53 \pm \\
0.01 \\
\end{array}$ & $\begin{array}{c}0.70 \pm \\
0.06\end{array}$ & $\begin{array}{c}0.80 \pm \\
0.01 \\
\end{array}$ & $\begin{array}{c}0.91 \pm \\
0.02 \\
\end{array}$ & $\begin{array}{c}0.95 \pm \\
0.02\end{array}$ & $\begin{array}{c}0.75 \pm \\
0.01 \\
\end{array}$ & $\begin{array}{c}0.88 \pm \\
0.04\end{array}$ & 0.79 & \\
\hline & 7 days & $\begin{array}{c}0.60 \pm \\
0.02\end{array}$ & $\begin{array}{c}0.73 \pm \\
0.07\end{array}$ & $\begin{array}{c}0.82 \pm \\
0.01\end{array}$ & $\begin{array}{c}0.88 \pm \\
0.06\end{array}$ & $\begin{array}{c}0.97 \pm \\
0.02\end{array}$ & $\begin{array}{c}0.75 \pm \\
0.01\end{array}$ & $\begin{array}{c}0.89 \pm \\
0.04\end{array}$ & 0.80 & \\
\hline & 14 days & $\begin{array}{c}0.63 \pm \\
0.02\end{array}$ & $\begin{array}{c}0.76 \pm \\
0.05\end{array}$ & $\begin{array}{c}0.84 \pm \\
0.02\end{array}$ & $\begin{array}{c}0.88 \pm \\
0.08\end{array}$ & $\begin{array}{c}0.98 \pm \\
0.02\end{array}$ & $\begin{array}{c}0.77 \pm \\
0.04\end{array}$ & $0.95 \pm 0.01$ & 0.83 & \\
\hline \multicolumn{2}{|c|}{ Mean } & 0.58 & 0.73 & 00.82 & 0.89 & 0.96 & 0.75 & 0.91 & & \\
\hline \multicolumn{2}{|c|}{ LSD } & \multicolumn{8}{|c|}{-} & \\
\hline \multirow[t]{3}{*}{$\begin{array}{c}\text { Chewiness } \\
(\mathrm{mJ})\end{array}$} & Fresh & $\begin{array}{c}17.31 \pm \\
0.45\end{array}$ & $\begin{array}{c}35.92 \pm \\
4.15\end{array}$ & $\begin{array}{c}73.03 \pm \\
0.05\end{array}$ & $\begin{array}{c}62.79 \pm \\
3.51\end{array}$ & $\begin{array}{c}96.35 \pm \\
0.93\end{array}$ & $\begin{array}{c}45.37 \pm \\
0.43\end{array}$ & $\begin{array}{c}96.94 \pm \\
6.73\end{array}$ & 61.10 & \multirow{3}{*}{2.63} \\
\hline & 7 days & $\begin{array}{c}22.65 \pm \\
1.41\end{array}$ & $\begin{array}{c}47.32 \pm \\
1.86\end{array}$ & $\begin{array}{c}94.59 \pm \\
1.81\end{array}$ & $\begin{array}{c}71.99 \pm \\
4.03\end{array}$ & $\begin{array}{c}111.55 \pm \\
5.24\end{array}$ & $\begin{array}{c}56.37 \pm \\
6.31\end{array}$ & $\begin{array}{c}111.65 \pm \\
6.41\end{array}$ & 73.73 & \\
\hline & 14 days & $\begin{array}{c}26.67 \pm \\
1.56\end{array}$ & $\begin{array}{c}56.65 \pm \\
1.76\end{array}$ & $\begin{array}{c}114.68 \pm \\
3.22\end{array}$ & $\begin{array}{c}78.95 \pm \\
4.50\end{array}$ & $\begin{array}{c}126.08 \pm \\
12.42\end{array}$ & $\begin{array}{c}63.88 \pm \\
6.19\end{array}$ & $\begin{array}{c}133.25 \pm \\
2.11\end{array}$ & 85.74 & \\
\hline \multicolumn{2}{|c|}{ Mean } & 22.21 & 46.63 & 94.10 & 71.24 & 111.32 & 55.21 & 113.95 & & \\
\hline \multicolumn{2}{|c|}{ LSD } & \multicolumn{8}{|c|}{3.72} & \\
\hline $\begin{array}{c}\text { Gummines } \\
\text { s (N) }\end{array}$ & Fresh & $\begin{array}{c}31.55 \pm \\
1.55\end{array}$ & $\begin{array}{c}51.63 \pm \\
1.24\end{array}$ & $\begin{array}{c}91.30 \pm \\
1.56\end{array}$ & $\begin{array}{c}69.36 \pm \\
2.26\end{array}$ & $\begin{array}{c}101.98 \pm \\
1.31\end{array}$ & $\begin{array}{c}60.90 \pm \\
0.00\end{array}$ & $\begin{array}{c}110.11 \pm \\
2.34\end{array}$ & 73.83 & \\
\hline
\end{tabular}


Improvement the properties of yoghurt using legumes to therapy

\begin{tabular}{|c|c|c|c|c|c|c|c|c|c|c|}
\hline & 7 days & $\begin{array}{c}37.82 \pm \\
1.34\end{array}$ & $\begin{array}{c}65.00 \pm \\
3.75\end{array}$ & $\begin{array}{c}115.35 \pm \\
0.21\end{array}$ & $\begin{array}{c}82.13 \pm \\
9.86\end{array}$ & \begin{tabular}{|c|}
$115.56 \pm$ \\
2.88 \\
\end{tabular} & $\begin{array}{c}75.09 \pm \\
7.00\end{array}$ & $\begin{array}{c}125.42 \pm \\
1.22\end{array}$ & 88.05 & \\
\hline & 14 days & $\begin{array}{c}41.60 \pm \\
2.55\end{array}$ & $\begin{array}{c}75.28 \pm \\
7.26\end{array}$ & $\begin{array}{c}137.34 \pm \\
0.37\end{array}$ & $\begin{array}{c}90.36 \pm \\
2.88\end{array}$ & $\begin{array}{c}129.20 \pm \\
9.93\end{array}$ & $\begin{array}{c}83.41 \pm \\
4.24\end{array}$ & $\begin{array}{c}140.30 \pm \\
4.31\end{array}$ & 99.64 & 2.48 \\
\hline \multicolumn{2}{|c|}{ Mean } & 36.99 & 63.97 & 114.66 & 80.61 & 115.58 & 73.13 & 125.27 & & \\
\hline \multicolumn{2}{|c|}{ LSD } & \multicolumn{8}{|c|}{3.51} & \\
\hline
\end{tabular}

See legend to table (1) for details.

\section{Organoleptic Properties}

The results of the evaluation of different yoghurt treatments through 14 days of the storage periods are shown in Table (5). Results indicated that addition of legumes improved organoleptic properties as compared with control yoghurt. Yoghurt produced using legumes increase score of colour in all treatments compared with control. Yoghurt fortified with kidney bean 6\% had the higher colour compared with other treatments. There was no significant difference in colour score in all treatments during storage period. Addition of legumes at different ratio improved the color, flavor, taste, viscosity and overall acceptation of yoghurt compared with control. For all level, as the storage period advanced the scoring point of organoleptic properties gradually decreased.

Table (5): Effect of type and ratio of legumes on sensory evaluation of set yoghurt during storage period

\begin{tabular}{|c|c|c|c|c|c|c|c|c|c|c|}
\hline $\begin{array}{c}\text { Organoleptic } \\
\text { Properties }\end{array}$ & $\begin{array}{c}\text { Storage } \\
\text { Period }\end{array}$ & C1 & T1a & T1b & T2a & $\mathrm{T} 2 \mathrm{~b}$ & T3a & T3b & Mean & LSD \\
\hline \multirow[t]{3}{*}{ Colour } & Fresh & $6.10 \pm 0.74$ & $8.40 \pm 0.84$ & $8.20 \pm 0.92$ & $8.00 \pm 0.67$ & $8.30 \pm 0.95$ & $8.00 \pm 0.94$ & $8.10 \pm 0.74$ & $7.87 \pm 1.09$ & \multirow[t]{3}{*}{---- } \\
\hline & 7 days & $5.90 \pm 0.88$ & $8.10 \pm 0.88$ & $7.90 \pm 0.88$ & $7.90 \pm 0.99$ & $8.20 \pm 0.92$ & $8.40 \pm 0.84$ & $8.00 \pm 1.05$ & $7.77 \pm 1.18$ & \\
\hline & 14 days & $5.91 \pm 0.83$ & $7.80 \pm 1.14$ & $8.20 \pm 0.92$ & $8.20 \pm 0.79$ & $8.10 \pm 0.57$ & $7.90 \pm 0.99$ & $8.10 \pm 0.74$ & $7.72 \pm 1.15$ & \\
\hline \multicolumn{2}{|l|}{ Mean } & $5.97 \pm 1.19$ & $8.10 \pm 0.96$ & $8.10 \pm 0.93$ & $8.03 \pm 0.83$ & $8.20 \pm 0.86$ & $8.10 \pm 0.79$ & $8.07 \pm 0.93$ & & \\
\hline \multicolumn{2}{|l|}{ LSD } & \multicolumn{9}{|c|}{0.68} \\
\hline \multirow[t]{3}{*}{ Taste } & Fresh & $5.10 \pm 0.88$ & $8.80 \pm 0.42$ & $8.50 \pm 0.85$ & $8.80 \pm 0.42$ & $8.80 \pm 0.42$ & $8.10 \pm 0.74$ & $8.30 \pm 0.95$ & $8.06 \pm 1.41$ & \multirow[t]{3}{*}{---} \\
\hline & 7 days & $5.00 \pm 0.94$ & $8.50 \pm 0.71$ & $8.50 \pm 0.71$ & $8.20 \pm 0.79$ & $8.60 \pm 0.52$ & $8.60 \pm 0.70$ & $8.70 \pm 0.48$ & $8.01 \pm 1.42$ & \\
\hline & 14 days & $5.00 \pm 0.89$ & $8.50 \pm 0.71$ & $8.50 \pm 0.85$ & $8.50 \pm 0.71$ & $8.90 \pm 0.32$ & $8.50 \pm 0.71$ & $8.30 \pm 0.95$ & $7.99 \pm 1.49$ & \\
\hline \multicolumn{2}{|c|}{ Mean } & $5.03 \pm 1.75$ & $8.60 \pm 0.62$ & $8.50 \pm 0.73$ & $8.50 \pm 0.77$ & $8.77 \pm 0.45$ & $8.40 \pm 0.68$ & $8.43 \pm 0.77$ & & \\
\hline \multicolumn{2}{|l|}{ LSD } & \multicolumn{9}{|c|}{0.56} \\
\hline \multirow[t]{3}{*}{ Flavor } & Fresh & $5.60 \pm 1.07$ & $8.40 \pm 0.52$ & $8.40 \pm 0.70$ & $8.10 \pm 0.88$ & $8.60 \pm 0.52$ & $8.30 \pm 0.82$ & $7.90 \pm 0.74$ & $7.90 \pm 1.22$ & \multirow[t]{3}{*}{---- } \\
\hline & 7 days & $5.70 \pm 1.16$ & $8.00 \pm 0.67$ & $7.90 \pm 0.74$ & $8.30 \pm 0.82$ & $8.40 \pm 0.52$ & $8.30 \pm 0.67$ & $8.20 \pm 0.63$ & $7.83 \pm 1.15$ & \\
\hline & 14 days & $5.18 \pm 1.17$ & $8.00 \pm 0.67$ & $8.40 \pm 0.70$ & $8.00 \pm 0.67$ & $8.30 \pm 0.48$ & $8.00 \pm 0.67$ & $7.90 \pm 0.74$ & $7.65 \pm 1.30$ & \\
\hline \multicolumn{2}{|c|}{ Mean } & $5.48 \pm 1.46$ & $8.13 \pm 0.63$ & $8.23 \pm 0.71$ & $8.13 \pm 0.77$ & $8.43 \pm 0.61$ & $8.20 \pm 0.65$ & $8.00 \pm 0.64$ & & \\
\hline \multicolumn{2}{|l|}{ LSD } & \multicolumn{9}{|c|}{0.59} \\
\hline \multirow{3}{*}{ Viscosity } & Fresh & $4.20 \pm 0.79$ & $8.80 \pm 0.42$ & $8.10 \pm 0.74$ & $8.10 \pm 0.74$ & $8.50 \pm 0.53$ & $8.40 \pm 0.70$ & $8.00 \pm 0.67$ & $7.73 \pm 1.61$ & \multirow[t]{3}{*}{--- } \\
\hline & 7 days & $4.30 \pm 0.82$ & $8.70 \pm 0.48$ & $8.20 \pm 0.79$ & $8.40 \pm 0.70$ & $8.60 \pm 0.52$ & $8.60 \pm 0.70$ & $8.50 \pm 0.71$ & $7.90 \pm 1.63$ & \\
\hline & 14 days & $4.09 \pm 0.83$ & $8.20 \pm 0.79$ & $8.10 \pm 0.74$ & $8.40 \pm 0.70$ & $8.20 \pm 0.42$ & $8.50 \pm 0.71$ & $8.00 \pm 0.67$ & $7.59 \pm 1.66$ & \\
\hline \multicolumn{2}{|c|}{ Mean } & $4.19 \pm 1.88$ & $8.57 \pm 0.63$ & $8.13 \pm 0.73$ & $8.30 \pm 0.71$ & $8.43 \pm 0.51$ & $8.50 \pm 0.63$ & $8.17 \pm 0.69$ & & \\
\hline \multicolumn{2}{|l|}{ LSD } & \multicolumn{9}{|c|}{0.53} \\
\hline & Fresh & $5.50 \pm 0.85$ & $8.60 \pm 0.52$ & $8.60 \pm 0.52$ & $8.60 \pm 0.70$ & $8.50 \pm 0.53$ & $8.30 \pm 0.82$ & $8.30 \pm 0.67$ & $8.06 \pm 1.24$ & --- \\
\hline
\end{tabular}




\begin{tabular}{|c|c|c|c|c|c|c|c|c|c|}
\hline \multirow{3}{*}{$\begin{array}{c}\text { overall } \\
\text { acceptabilit } \\
y\end{array}$} & 7 days & $5.60 \pm 0.97$ & $8.20 \pm 0.63$ & $8.10 \pm 0.74$ & $8.20 \pm 0.79$ & $8.40 \pm 0.52$ & $8.60 \pm 0.52$ & $8.30 \pm 0.67$ & $7.91 \pm 1.18$ \\
\hline & 14 days & & & & & & & & $7.87 \pm 1.25$ \\
\hline & & $5.55 \pm 1.04$ & $7.90 \pm 0.74$ & $8.60 \pm 0.52$ & $8.40 \pm 0.70$ & $8.50 \pm 0.71$ & $8.10 \pm 0.74$ & $8.30 \pm 0.67$ & \\
\hline \multicolumn{2}{|c|}{ Mean } & $5.55 \pm 1.51$ & $8.23 \pm 0.68$ & $8.43 \pm 0.68$ & $8.40 \pm 0.68$ & $8.47 \pm 0.57$ & $8.33 \pm 0.68$ & $8.30 \pm 0.65$ & \\
\hline \multicolumn{2}{|c|}{ LSD } & \multicolumn{8}{|c|}{0.54} \\
\hline
\end{tabular}

See legend to table (1) for details.

\section{Conclusion}

The obtained results suggest possibility of making a good quality high protein and calorie yoghurt with the use of legumes. Results also indicated that $6 \%$ kidney bean and $3 \%$ chickpea flour may be useful ingredient for production of yoghurt without adversely effect on the properties of the product.

\section{REFERENCES}

Abrahamsson, L., A. Andersson, W. Becker and G. Nilsson (2006). Human Nutrition and Applied Nutrition. Stockholm: Liber AB.

Adeyeba, A.O. (2014). Utilization of Legumes in the Tropics. Journal of Biology, Agriculture and Healthcare.4: 2224-3208.

Anstead, G.M., B. Chandrasekar, W. Zhao, J. Yang, L.E. Perez and P.C. Melby (2001). Malnutrition alters the innate immune response and increases early vascularization following Leishmania donovani infection. Infection and Immunity, 69:4709 - 4718.

AOAC. (2000). Official Methods of Analysis. Association of official analytical chemists. 17th Ed., Washington DC, USA.

Ashworth, A. and E. Ferguson (2009). Dietary counseling in the management of moderate malnourishment in children. Food and Nutrition Bulletin, 30:405-433.

Bourne, MC. (1978). Texture profile analysis. Food Technology., 62:330366

Bravo, L., P. Siddhuraju and F. SauraCalixto (1999). Composition of underexploited Indian pulses, comparison with common legumes. Food Chemistry, 64: 185-192.

Codex Alimentarius (2003). Codex Standards for Fermented Milk. Codex Stan. 243, FAO, Rome, Italy.

Dean, C., Scholl, F.G. Choih, J. et al. (2003). Neurexin mediates the assembly of presynaptic terminals. Nature Neuroscience, 6:708-716.

El-Beltagy, A. (1996). Effect of home traditional methods on quality aspected of some legumes. M.Sc. Thesis, Faculty of Agriculture, Menofiya University, Shibin El-Kom, Egypt .

Ernest, N., P. Akpan and E. Uko (2013). Reduced levels of some iron parameters of protein energy malnourished children in Calabar, Nigeria. Journal of Biology, Agricultural and Healthcare, 3:114120.

Ezzati, M., A.D. Lopez, A. Rodgers, H. S. Vander and C.J. Murray (2002). Selected major risk factors and global and regional burden of disease. US National Library of Medicine National Institutes of Health. 360:1347-1360.

Lal, S.N.D., C.J. O. Connor and L. Eyres (2006). Application of emulsifiers । stabilizers in dairy products of high rheology. Advance in Colloid and Interface Science, 123-126,433-437.

Ling, E.R. (1963). A Textbook of Dairy Chemistry. Vol. 11, Practical, 3rd Ed., Chapman and Hall, London, UK.

Makanjuola, O.M. (2012). production and quality evaluation of soy-corn yoghurt. Journal of Food Science and Technology 4: 130-134 
Mckinley, M. C. (2005). The nutrition and health benefits of yoghurt. International Journal of Dairy Technology.

Oke, D. B., O. O. Tewe and S. L. Fetuga (1995). The nutrient composition of some cowpea varieties. Nigerian Journal of Animal Production.22: 3236

Reddy, N. R., M. D. Pierson, S. K. Sathe and D. K. Salunkhe (1985). Dry bean tannins - a review of Nutritional implications. Journal of the American Oil Chemists Society. $62: 541-549$.

Rehman, Z.U. and W. H. Shah (2004). Domestic processing effects on someinsoluble dietary fibre components of various food legumes. Food Chemistry, 87: 613-617

Rizwana, B., M. Sadiq Butt, M. Tauseef Sultan, S. Farhan and N. Rabia (2015). Protein-energy malnutrition: a risk factor for various ailments. Critical Reviews in Food Science and Nutrition, 55:242-253.

Sandoval-castilla, O., C. LobatoCallerors, E. Aguirre-Mandujano and R. J. Vernon-Carter (2004). Microstructure and texture of yoghurt as influence by fat replacers. International Dairy Journal, 14:151159.

Shaker, R. R., B. Abu-Jdayil and R.Y. Jumah (2000). Rheological properties of a concentrated fermented product. Labneh, produced from bovine milk: effect of production method.
International Journal of Food properties, 5: 667-679.

Szczesniak, A.S., M.A. Brandt and H.H. Friedman (1963). Development of standard rating scales for mechanical parameters of texture and correlation between the objective and sensory methods of texture evaluation. Journal of Food Science, 28: 397.

Tamime, A.Y. (2006). Fermented Milks. Blackwell Science Ltd, Oxford, England.

Tamime, A. Y. and R. K. Robinison (1999). Yoghurt: Science and Technology, ${ }^{2}$ nd ed. Wood head Publishing Ltd., Cambridge, UK.

Une, L. and S. Gupta (2013). Micronutrients and antioxidant status in children with protein energy malnutrition. Asian Journal of Biomedical Pharmaceutical Science, 3: 38 - 40.

Yin, S.W., C.H. Tang, Q.B. Wen, X.Q. Yang and L. Li (2008). Functional properties and in vitrotrypsin digestibility of red kidney bean (Phaseolus vulgaris L.) protein isolate: effect ofhigh-pressure treatment. Food Chemistry. 110: 938945

Zare, F., C.P. Champagne, B.K. Simpson, V. Orsat and J.I. Boye (2011). Effect of the addition of pulse ingredients to milk on acid production by probiotic and yoghurt starter cultures .LWTfood science and technology.45:155160. 
تحسين خصائص اليوجورث باستخدام البقوليات لعلاج سوء التغذية الناتج عن نقص البروتين في الاطفال

ابتسام محمد فايد بلر (1) ، دينا احمد مرتضى عامر (1) ، محمد يحيي علي الهواري(1) ، أحمد محمد عبد العال نعيم(2)

(1) - قسم علوم وتكنولوجيا الاغذيه- كلية الزراعة- جامعة طنطا. (2) - قسم طب الأطفال - كلية الطب - جامعة طنطا.

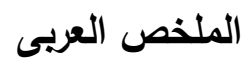

تهدف هذه الدراسة الى تحسين خصائص اليوجورت باستخدام أنواع مختلفة من البقوليات بنسب مئوية مختلفة مثل

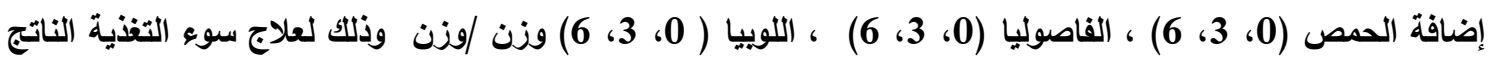

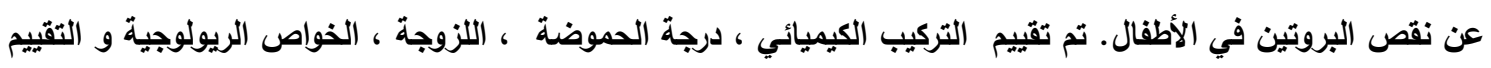

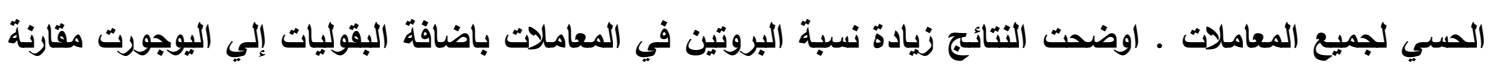
بالكتترول ـ اليوجورت المنتج باضافه 6\% من الفاصوليا يحتوي علي اعلي نسبة بروتين مقارنة بباقي المعاملات .

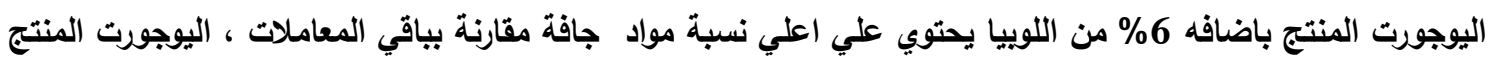

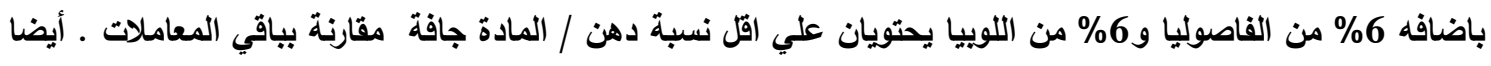

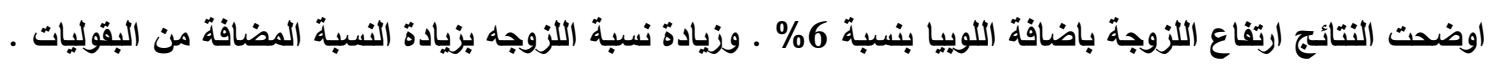

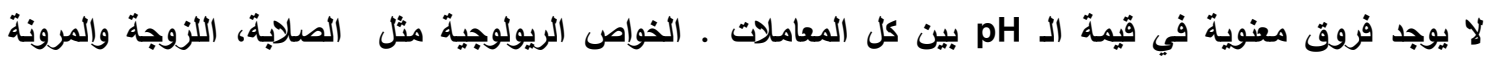

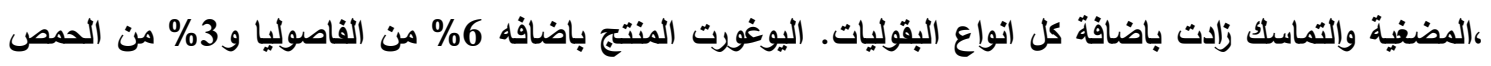
كان له أفضل الخواص الريولوجية و الحسية مقارنة بباقي المعاملات. 
Improvement the properties of yoghurt using legumes to therapy ................

أسماء السادة المحكمين

أ.د/ سامى عبدالرحمن كلية الزراعة - جامعة المنوفية

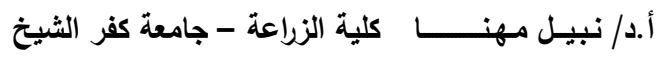

